\title{
Stability, control and discretization for a smoking model
}

\author{
M Ozair ${ }^{*}$, Q Din ${ }^{2}$, T Donchev ${ }^{3}$ and T Hussain ${ }^{1}$ \\ ${ }^{1}$ Department of Mathematics, COMSATS University Islamabad, Attock Campus, Attock, Pakistan. \\ ${ }^{2}$ Department of Mathematics, University of Poonch Rawalakot, Azad Kashmir, Pakistan. \\ ${ }^{3}$ Department of Mathematics, University of Architecture and Civil Engineering, Sofia 1046, Bulgaria.
}

\begin{abstract}
In this paper, the deterministic model of smoking consisting of five classes, has been qualitatively analysed. Explicit formula for the reproduction number has been obtained. Equilibria have been found and their global asymptotic stability has been discussed. Method of matrix theoretic, with the Perron eigenvectors, is used to get the global asymptotic stability of smoking-free equilibrium. It is shown that unique endemic equilibrium is globally asymptotically stable by using graph theoretic approach. To know the important factors, through which the disease spreads rapidly, sensitivity analysis of basic reproduction number and endemic level of smokers has been performed. This sensitivity analysis urged to modify the existing problem by inserting two controls namely prevention and treatment. Optimal control problem has been designed on the basis of sensitivity analysis. The existence of controls has been proved analytically and numerically it is shown that these applied controls significantly reduce the number of smokers. Moreover, a dynamically consistent nonstandard difference scheme is implemented to obtain a 5-dimensional discretetime smoking model. Necessary and sufficient conditions are obtained for local dynamics of equilibria for discrete-time smoking model. Fourth-order implicit Runge--Kutta method is implemented to see the effectiveness of proposed nonstandard difference scheme. Numerical simulations have been done for the verification of the analytical results.
\end{abstract}

Keywords: Epidemic model, graph-theoretic method, nonstandard difference scheme, Perron eigenvector, sensitivity, stability.

\section{INTRODUCTION}

Smoking kills more than 7 million people each year throughout the world and with the passage of time this number is increasing (WHO, 2017). According to the World Health Organization (WHO, 2009), use of tobacco either kills or weakens the people in their healthiest and fruitful ages and it assects the economy of the country. Smoking is the principal cause of life threatening diseases, such as lungs cancer, oesophagus, heart and respiratory diseases and tuberculosis (Lahrouz et al., 2011). According to Surgeon General Report in 1991, a person starts to smoke at average age of 14.5 years and become daily smoker at 17.7 (on average) years. Statistical data from 1975 to 1992 shows that the key time to start the use of tobacco is the teenage years (Johnston et al., 1992). Some community based factors all so contribute for the smoking such as smoking habits of relatives or associates.

Mathematical modelling is an effective tool to control health related issues. History of mathematical modelling goes back to pioneer work of Bernoulli in 1760 which is about dynamics of smallpox (Bernoulli, 1760), then Kermack and Mckendrick (1927; 1932; 1933) and modern literature (Anderson \& May, 1982 and 1991,

\footnotetext{
* Corresponding author (ozairmuhammad@gmail.com; (iD https://orcid.org/0000-0001-5964-4351)
} 
Brauer \& Chavez, 2001). The use of mathematical modelling for the human community behaviour is rare. Castillo-Garsow et al., presented a mathematical model on quitting of smoking in 1997. They proposed a nonlinear system of differential equations having three groups of population: potential smokers, smokers and quitters. Sharomi and Gumel presented a model in which they divided quitter's class into temporary quitters $\left(Q_{t}\right)$ and permanent quitters $\left(Q_{p}\right)$ (Sharomi \& Gumel, 2008). Zaman extended the model of Castillo-Garsow et al. by adding occasional smoker's class and then studied it qualitatively (Zaman, 2011). Zeb et al., discussed the square root dynamics of a quitting smoking model (Zeb et al., 2012). Recent models on smoking were presented by (Verma \& Agarwal; 2015; Din et al., 2016; Awan et al., 2017; Zeb et al., 2018; Shaheen et al., 2018; Verma V. \& Bhadauria A., 2019).

\section{METHODOLOGY}

We modified the model given in (Zeb et al., 2012) in which the authors considered only three classes namely, potential smokers, smokers and quitters. We extended this model by considering temporary and permanent quitters and humans having some disease due to smoking. We also include the following features:

- Temporary quitters again become smokers.

- A class of people who get any disease due to smoking.

- Disease related death rate.

\section{Construction of model}

Let $P$ denotes the people who are susceptible to smoke but not smokers. $S$ represents the smokers, $X$ is the number of persons who quit smoking for a time. People who are permanent quitters lie in the class $Y$ and if the smokers get some illness due to smoking then they transfer into class $Z$. The sum of population is denoted by $N$ which is considered as the entire population at any time $t$.

We assume that susceptible people grow at the rate $\alpha$. When susceptible people have a physical interaction with smokers then they move from class $P$ to $S$ with a rate $\beta$. $\gamma$ is the rate at which people die naturally. The smokers give up smoking temporarily and forever at the rate of $\delta(1-\eta)$ and $\delta \eta$, respectively. The parameter $\eta$ lies between 0 and 1 . Those people who get any disease because of smoking move from $S$ to $Z$ at the rate $\varepsilon$. Temporary quitters get back to class of smokers with rate $\zeta$. The people die, because of smoking associated disease, at the rate $\vartheta$. We can represent the model with the following flow diagram:

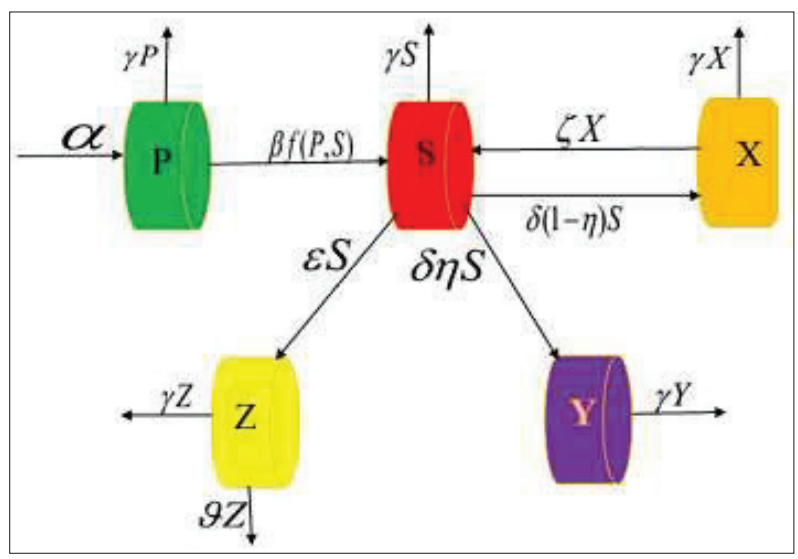

Figure 1: Flow sheet of smoking disease model.

Mathematical representation of model is given below:

$$
\begin{aligned}
& \frac{d P}{d t}=\& \alpha-\beta P S-\gamma P, \\
& \frac{d S}{d t}=\& \beta P S-\gamma S-\delta S-\varepsilon S+\zeta X, \\
& \frac{d X}{d t}=\& \delta(1-\eta) S-(\gamma+\zeta) X \\
& \frac{d Y}{d t}=\& \delta \eta S-\gamma Y, \\
& \frac{d Z}{d t}=\& \varepsilon S-(\gamma+\vartheta) Z .
\end{aligned}
$$

By adding all the differential equations of system of equations (1-5), and solving the resultant differential equation, we have

$$
N(t)=P(t)+S(t)+X(t)+Y(t)+Z(t) \leq \frac{\alpha}{\gamma},
$$

Thus, we can write the feasible region for system of equations (1-5) as follows

$$
\Omega=\left\{(P, S, X, Y, Z) \in R_{+}^{5}, N(t) \leq \frac{\alpha}{\gamma}\right\} .
$$




\section{RESULTS AND DISCUSSION}

\section{Smoke free equilibrium}

To find the smoke free equilibrium of (1-5), we will take all derivatives and $S(t)=0$. Then, we get

$$
\begin{aligned}
\alpha-\gamma P & =0, \\
P & =\frac{\alpha}{\gamma},
\end{aligned}
$$

and the following equilibrium point

$$
E_{0}\left(P_{0}, S_{0}, X_{0}, Y_{0}, Z_{0}\right)=E_{0}\left(\frac{\alpha}{\gamma}, 0,0,0,0\right) .
$$

\section{Local Stability of smoke-free equilibrium}

In this section, with the help of threshold parameter $R_{0}$, we study that smoke free equilibrium point is locally stable. Driessche and Watmough, introduced a method to find $R_{0}$, this method is known as "next generation matrix method" (Driessche \& Watmough, 2002). By following this method, the matrices of new infection and remaining terms are as follows

$$
\begin{aligned}
F & =\left(\begin{array}{cccc}
\beta P_{0} & 0 & 0 & 0 \\
0 & 0 & 0 & 0 \\
0 & 0 & 0 & 0 \\
0 & 0 & 0 & 0
\end{array}\right) \\
V & =\left(\begin{array}{cccc}
\delta+\gamma+\varepsilon & -\zeta & 0 & 0 \\
\eta \delta-\delta & \gamma+\zeta & 0 & 0 \\
-\delta \eta & 0 & \gamma & 0 \\
-\varepsilon & 0 & 0 & (\gamma+\vartheta)
\end{array}\right)
\end{aligned}
$$

Then the greatest eigenvalue, in magnitude, of the matrix $F V^{-1}$ is called the threshold parameter $R_{0}$ and is given by

$$
R_{0}=\frac{\beta \frac{\alpha}{\gamma}}{(\delta+\varepsilon+\gamma)}+\frac{\zeta \delta(1-\eta)}{(\gamma+\zeta)(\delta+\varepsilon+\gamma)} .
$$

One can know, with the help of $R_{0}$, about the new smoker produced by the single smoker in its smoking period. If $R_{0}<1$, then it tells that there is no smoking in the population but if its value is greater than one then smoking can expand in the community. Driessche and Watmough, set up a result which is given below (Driessche \& Watmough, 2002),

Theorem 1. If $R_{0}<1$, then smoke free equilibrium point is locally asymptotically stable otherwise unstable.

\section{Global stability of smoke-free equilibrium}

In order to show that smoke free equilibrium point is globally stable when $R_{0} \leq 1$, we use the Lyapunov function in the form of Perron eigenvectors.

Theorem 2. The smoke-free equilibrium point of the system of equations (1-5) is stable globally when $R_{0} \leq 1$.

Proof. To show this, following Lyapunove function has been introduced:

$$
L(t)=\omega^{T} V^{-1} x,
$$

where $\omega^{T}=\left(\begin{array}{llll}1 & \frac{\zeta}{\beta P_{0}} & 0 & 0\end{array}\right)$ is left eigenvector of matrix $V^{-1} F$ and $x=(S, X, Y, Z)^{T}$.

By simple calculation we obtain

$L(t)=\left(\frac{R_{0}}{\beta P_{0}}\right)\left[S+\left(\frac{\zeta(\gamma+\delta+\varepsilon)-\zeta \beta P_{0}}{\beta P_{0}(\gamma+\zeta)+\zeta \delta(1-\eta)}\right) X\right]$,

and

$L^{\prime}(t)=$

$$
\begin{gathered}
\left(\frac{R_{0}}{\beta P_{0}}\right)\left[\begin{array}{c}
\left(\beta P-(\gamma+\delta+\varepsilon)+\frac{\zeta \delta(1-\eta)}{(\gamma+\zeta) R_{0}}-\frac{\beta P_{0} \zeta \delta(1-\eta)}{(\delta+\varepsilon+\gamma)(\gamma+\zeta) R_{0}}\right) S \\
+\left(\zeta-\frac{\zeta}{R_{0}}+\frac{\zeta \beta P_{0}}{(\delta+\varepsilon+\gamma) R_{0}}\right) X
\end{array}\right] \\
L^{\prime}(t) \leq\left(\frac{R_{0}}{\beta P_{0}}\right)\left[\beta P_{0}\left(1-\frac{1}{R_{0}}\right) S-\frac{\beta P_{0} \zeta \delta(1-\eta)}{(\delta+\varepsilon+\gamma)(\gamma+\zeta) R_{0}} S\right. \\
\left.+\zeta\left(2-\frac{1}{R_{0}}\right) X-\frac{\zeta^{2} \delta(1-\eta)}{(\delta+\varepsilon+\gamma)(\gamma+\zeta) R_{0}} X\right]
\end{gathered}
$$

Furthermore $L^{\prime}(t)=0$ implies $S=0$ and $X=0$. Thus, the set $\left\{E_{0}\right\}$ is the only singleton set which contains both $S=0$ and $X=0$. Hence, we have the result by LaSalle's invariant principle (LaSalle, 1987).

\section{Endemic equilibrium}

To find the endemic equilibrium point $E^{*}=\left(Z^{*}, Y^{*}, X^{*}, P^{*}, S^{*}\right)$, we will have to set the derivatives of nonlinear system of differential equations (1-5) equal to zero then after calculations, we have

$$
Z^{*}=\frac{\varepsilon}{(\gamma+\vartheta)} S^{*}, Y^{*}=\frac{\delta \eta}{\gamma} S^{*}
$$




$$
\begin{aligned}
X^{*} & =\frac{\delta-\eta \delta}{(\gamma+\zeta)} S^{*}, P^{*}=\frac{\alpha}{\left(\beta S^{*}+\gamma\right)}, \\
S^{*} & =\frac{(\gamma+\delta+\varepsilon) \gamma(\gamma+\zeta)\left[R_{0}-1\right]}{\beta[(\gamma+\delta+\varepsilon)(\gamma+\zeta)-\zeta \delta(1-\eta)]} .
\end{aligned}
$$

\section{Global stability of endemic equilibrium}

By using graph theoretic approach (Harary, 1969; West, 1996), we will show that our endemic equilibrium point is globally stable. As stated in (Shuai \& Driessche, 2013), following are some fundamental definitions associated with this approach.

A directed graph, or digraph, is a graph $G$, which contains vertices $(V(G))$, edges $E(G)$, and a mapping that allocates an ordered couple of vertices $(i, j)$ to each edge. If an edge is represented by a pair $(i, j)$ then it means that this edge is directed from $i$ to $j$. The edges $(l, i), l \in V$, and $(i, l), l \in V$, represent the indegree $d_{G}^{-}(i)$ and outdegree $d_{G}^{+}(i)$ of any vertex $i$. We say that $H$ is a sub digraph if it is the spanning set of $G$ contains the same sets of vertex. A weighted digraph $\mathrm{H}$ is the graph if a weight has been allocated to its each edge and if we multiply all the weights allocated to every edges of a sub digraph $(H)$ then that is called weight $W(H)$ of a sub digraph $H$.

If indegree is zero for only one vertex and it is equal to for the rest of vertices of a subdigraph $T$ of $G$ then that sub digraph is called a tree. If a sub digraph of $G$ having vertices represented by $i_{1}, i_{2}, i_{3}, \cdots, i_{k}$ so that its edges having the form $\left(i_{q}, i_{q+1}\right), q=1,2,3, \cdots, i_{k-1}$, then that sub digraph is known as a directed path $P$. Furthermore, the sub digraph, which is established from a path $P$ by including the edge $\left(i_{k}, i_{1}\right)$, is known as a directed cycle $C$. A weighted digraph $(G, A)$ is a digraph with number of vertices $n$, where $A=\left(a_{i j}\right)$ is the weighted matrix with $a_{i j}>0$ denotes the weight of edge $(j, i)$ if it exists and 0 otherwise. If a directed way, which starts from $i$ and ends at $j$ for any couple of different vertices $j$, exists then that digraph is called strongly connected. An irreducible weight matrix $A$ always implies a strongly connected weighted digraph $(G, A)$ and vice versa. We can define the Laplacian matrix $L=\left(l_{i j}\right)$ of $(G, A)$ in the following way

$$
l_{i j}=\left\{\begin{array}{c}
-a_{i j}, i \neq j, \\
\sum_{i \neq k} a_{i k}, i=k .
\end{array}\right.
$$

Lemma 3. (Kirchhoff's matrix tree theorem) We suppose that cofactor of $l_{i i}$ is represented by $c_{i}$ and let $T$ represents the spanning tree of $(G, S)$. If, $T_{i}$ is the set of spanning trees of $(G, S)$ and roots of these trees are at vertex $i$. Then $c_{i}=\sum_{T \in T_{i}} W(T) i=1,2,3, \cdots, n$ for $n \geq 2$. Furthermore, a weighted digraph, $(G, A)$, implies $c_{i}>0$. for i varies from 1 to $\mathrm{n}$ that is $1 \leq i \leq n$.

We have some more results according to the arguments given in (Shuai, 2010, Shuai \& Driessche, 2013).

Lemma 4. Suppose that $F_{i}(X)$ be a set of functions such that $X \in R^{m}$ that is $X=\left(x_{1}, x_{2}, x_{3}, \cdots, x_{m}\right)$ and let $c_{i}$ as stated in above Lemma $K$ then we have the result $\sum_{i, j=1}^{n} c_{i} a_{i j} F_{i}(X)=\sum_{i, j=1}^{n} c_{i} a_{i j} F_{j}(X)$

Lemma 5. Let us suppose that for any $1 \leq i, j \leq n, a_{i j}>0$, and $d_{G}^{+}(j)=1$. Then as given in (Shuai \& Driessche, 2013), $c_{i} a_{i j}=\sum_{k=1}^{n} c_{j} a_{j k}$ where is stated in Lamma 3.

Theorem 6. For $R_{0}>1$, the endemic equilibrium point $E^{*}$ is globally asymptotically stable.

$V_{1}=P-P^{*}-\ln \frac{P}{P^{*}}, V_{2}=S-S^{*}-\ln \frac{S}{S^{*}}$,

$V_{3}=X-X^{*}-\ln \frac{X}{X^{*}}, V_{4}=Y-Y^{*}-\ln \frac{Y}{Y^{*}}$,

$V_{5}=Z-Z^{*}-\ln \frac{Z}{Z^{*}}$.

Proof. The following Lyapunov functions are constructed:

$$
\begin{gathered}
V_{1}^{\prime} \leq \beta P^{*} S^{*}\left(\frac{S}{S^{*}}-\ln \frac{S}{S^{*}}+\ln \frac{S}{S^{*}}-\ln \frac{P}{P^{*}}-\frac{P S}{P^{*} S^{*}}\right)=: a_{12} G_{12}, \\
V_{2}^{\prime} \leq \beta P^{*} S^{*}\left(\frac{P S}{P^{*} S^{*}}-\ln \frac{P S}{P^{*} S^{*}}+\ln \frac{P}{P^{*}}-\frac{P}{P^{*}}\right)+ \\
\zeta X^{*}\left(\frac{X}{X^{*}}-\ln \frac{X}{X^{*}}-\frac{X}{X^{*}} \frac{S^{*}}{S}+\ln \frac{X}{X^{*}} \frac{S^{*}}{S}\right)
\end{gathered}
$$

Taking time derivatives we have

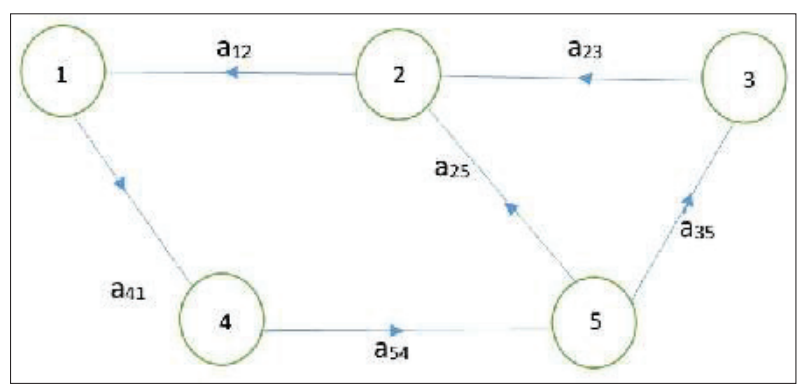

Figure 2: Weighted-diagraph constructed for system of equations (1-5) 


$$
\begin{array}{ll}
V_{1}^{\prime} \leq \beta P^{*} S^{*}\left(\frac{S}{S^{*}}-\ln \frac{S}{S^{*}}+\ln \frac{S}{S^{*}}-\ln \frac{P}{P^{*}}-\frac{P S}{P^{*} S^{*}}\right)=: a_{12} G_{12}, & V_{3}^{\prime} \leq \delta(1-\eta) S^{*}\left(\frac{S}{S^{*}}-\frac{S X^{*}}{S^{*} X}+\ln \frac{S X^{*}}{S^{*} X}-\ln \frac{S}{S^{*}}\right)=: a_{35} G_{35}, \\
V_{2}^{\prime} \leq \beta P^{*} S^{*}\left(\frac{P S}{P^{*} S^{*}}-\ln \frac{P S}{P^{*} S^{*}}+\ln \frac{P}{P^{*}}-\frac{P}{P^{*}}\right)+ & V_{4}^{\prime} \leq \delta \eta S^{*}\left(\frac{S}{S^{*}}-\ln \frac{S}{S^{*}}-\frac{S Y^{*}}{S^{*} Y}+\ln \frac{S Y^{*}}{S^{*} Y}\right)=: a_{41} G_{41}, \\
\zeta X^{*}\left(\frac{X}{X^{*}}-\ln \frac{X}{X^{*}}-\frac{X}{X^{*}} \frac{S^{*}}{S}+\ln \frac{X}{X^{*}} \frac{S^{*}}{S}\right)=: a_{23} G_{23}+a_{25} G_{25}, & V_{5}^{\prime} \leq \epsilon S^{*}\left(\frac{S}{S^{*}}-\ln \frac{S}{S^{*}}-\frac{S Z^{*}}{S^{*} Z}+\ln \frac{S Z^{*}}{S^{*} Z}\right)=: a_{54} G_{54} .
\end{array}
$$

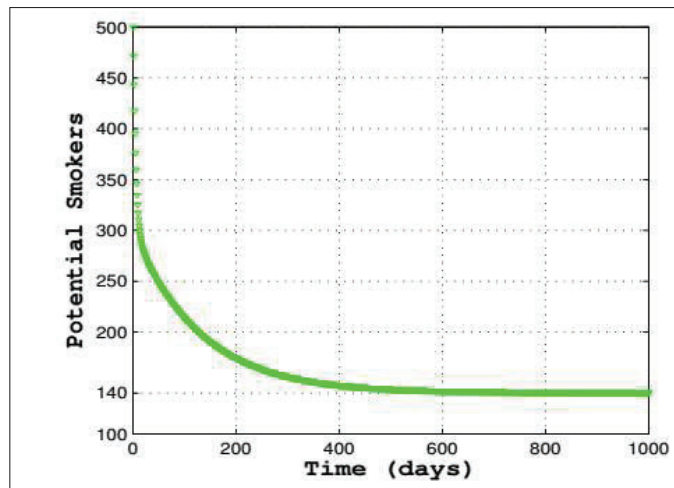

(a)

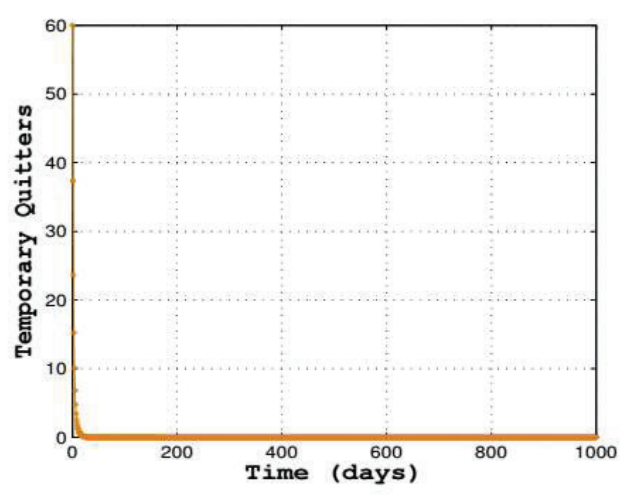

(c)

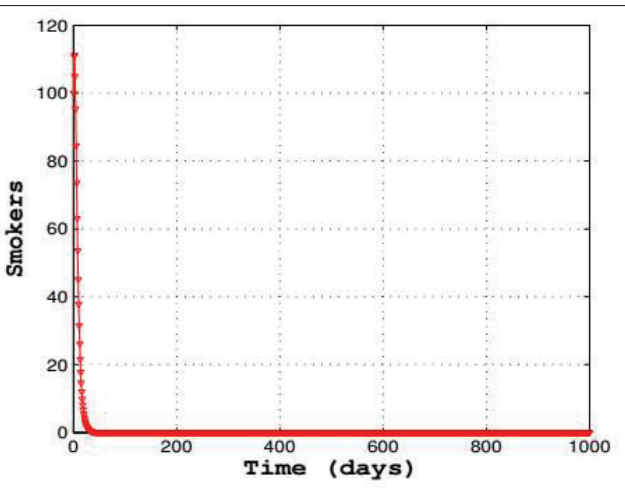

(b)

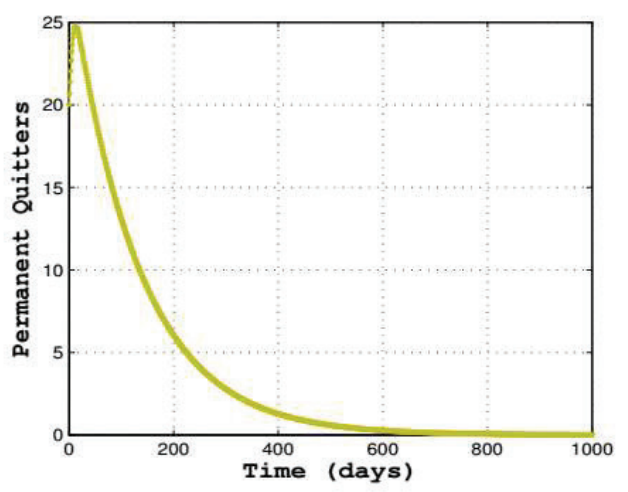

(d)

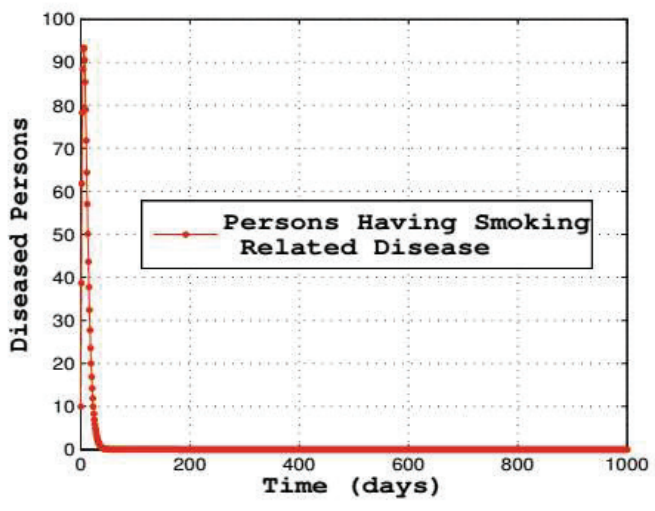

(e)

Figure 3: The Population approaches disease-free equilibrium when $R_{0}<1$. 
From figure 2, one can see that there are five vertices and two cycles for the associated weighted digraph. One can write for each cycle $G_{12}+G_{41}+G_{54}+G_{25}=0$ and $G_{12}+G_{41}+G_{54}+G_{35}+G_{23}=0$. As stated in Theorem of (Shuai and Driessche, 2013), $c_{i}, 1 \leq i \leq 5$, exists for which we have a Lyapunov function for the given system of equations (1-5) has the form $V=\sum_{i=1}^{5} c_{i} V_{i}$. We have following relations between $c_{i}$ 's

$d_{G}^{+}(1)=1, \quad d_{G}^{+}(2)=1, \quad d_{G}^{+}(3)=1, \quad d_{G}^{+}(4)=1$, these relations are stated in Theorem and of (Shuai \& Driessche, 2013). And we can write from above relations respectively $c_{4} a_{41}=c_{1} a_{12}$, $c_{1} a_{12}=c_{2}\left(a_{23}+a_{25}\right), c_{2} a_{23}=c_{3} a_{35}, c_{5} a_{54}=c_{4} a_{41}$. Therefore $\quad V=\sum_{i=1}^{5} c_{i} V_{i}=V_{1}+\frac{\beta P^{*} S^{*}}{\left(\beta P^{*} S^{*}+\zeta X^{*}\right)} V_{2}$ $+\frac{\left(\beta P^{*}\right)^{2} S^{*}}{\delta(1-\eta)\left(\beta P^{*} S^{*}+\zeta X^{*}\right)} V_{3}+\frac{\beta P^{*}}{\delta \eta} V_{4}+\frac{\beta P^{*}}{\epsilon} V_{5}$. Thus, it can be easily proved that the only invariant singleton set is the set $\left\{E^{*}\right\}$ in $(\Omega)$ where $V^{\prime}=0$, hence we have the result. Now we solve the model numerically. Figure 3 depicts that the population meets the disease-free equilibrium point for $R_{0}<1$, while Figure 4 represents that the population is approaching to endemic level for $R_{0}>1$.

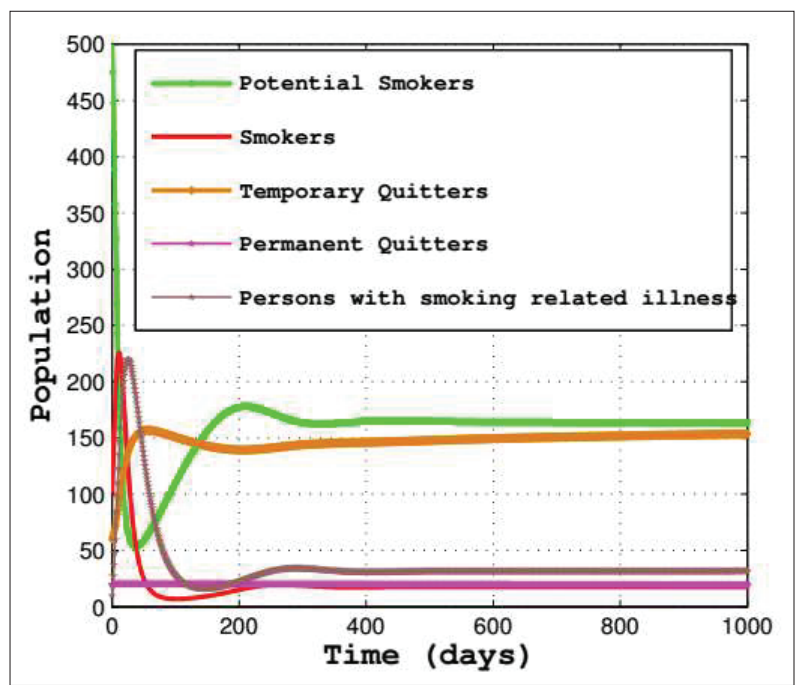

Figure 4: The population approaches endemic equilibrium when $R_{0}>1$.

\section{Sensitivity analysis}

We are interested to analyse those parameters due to which disease spread more rapidly from infectious person to susceptible. Destruction as a result of smoking can be decreased by this way. Threshold parameter $R_{0}$ is responsible for the spread of disease and endemic equilibrium point tells the persistence of disease. We know that the basic reproduction number measures the average number of secondary infections produced by an infectious individual during its infectious period. Therefore it is important to make the reproduction number below unity for the complete eradication of the disease. For this purpose, we identify the most influential factor that plays vital role in order to reduce the basic reproduction number below unity. However, it is impossible to eradicate the smoking menace completely from the community so we also focus on the possible reduction of number of smokers in the community. The people of group are the main resources due to which disease spread. The definition given below will be used to find the sensitive indices of smokers and $R_{0}$.

Definition 7. If, we have a variable and it has a parameter hen we can find the normalized forward sensitivity of $y$ W. r. t. $t$ with the help of formula as follows $\gamma_{t}^{y}=\frac{\partial y}{\partial t} \times \frac{t}{y}$. The sensitivity indices with respect to the model parameters are presented in the following Table 1.

Table 1: The sensitivity indices with respect to the model parameters.

\begin{tabular}{cll}
\hline Parameter & \multicolumn{1}{c}{$R_{0}$} & \multicolumn{1}{c}{$\mathrm{S}$} \\
\hline$\alpha$ & 0.999055 & 1.00438 \\
$\beta$ & 0.999055 & 0.00437816 \\
$\delta$ & -0.180802 & -0.00425981 \\
$\varepsilon$ & -0.817859 & -0.999636 \\
$\eta$ & $-9.44729 \times 10^{-10}$ & $-2.17881 \times 10^{-7}$ \\
$\gamma$ & 0.999468 & -0.00903861 \\
$\zeta$ & $1.81153 \times 10^{-5}$ & 0.00417791 \\
\hline
\end{tabular}

The sensitivity of threshold parameter $\mathrm{R}_{0}$ and the endemic level of $S^{*}$ reveals that the most sensitive parameters for the reproduction number are $\alpha, \beta, \delta, \gamma, \varepsilon$ and for the endemic level of smokers are $\alpha, \varepsilon$. Sensitivity indices of $\alpha$ and $\gamma$ will not be under consideration because these are birth and natural death rates, respectively. The remaining parameters are $\beta, \delta$ and $\varepsilon$. We can observe that $\mathrm{R}_{0}$ and $S^{*}$ are directly related to $\beta$ and inversely related to $\delta$ and $\varepsilon . R_{0}$ as a function of $\beta, \delta$ and $\varepsilon$ is shown in figure (5). From this figure we can observe that $R_{0}$ is readily increasing with respect to $\beta$ and decreasing with respect to $\delta$ and $\varepsilon$. These sensitive parameters suggest that we can control the disease by starting the campaign through media transmission in which we briefly explain how the population indulging in this addiction ultimately die. This death occurs due to smoking related illness. 


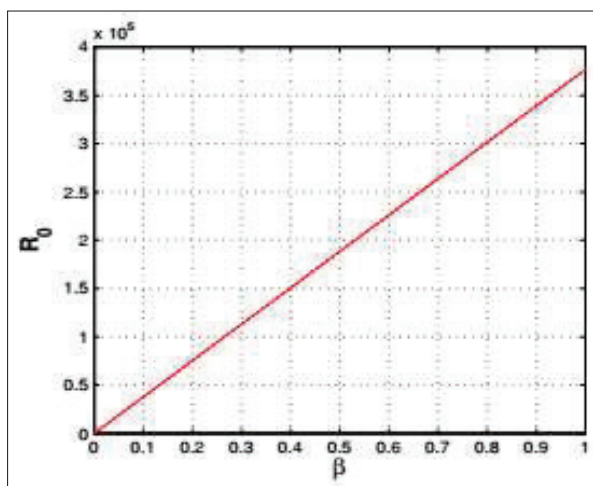

(a)

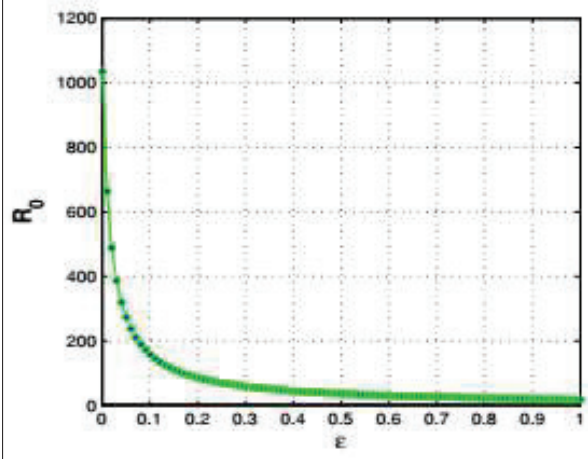

(c)

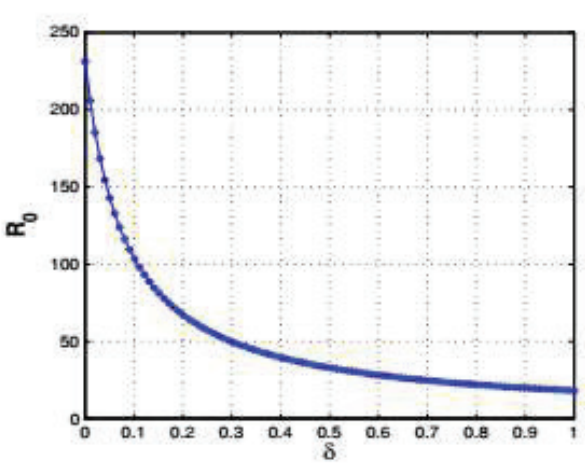

(b)

Figure 5: Variation in the value of $R_{0}$ with respect to $\beta, \delta$ and $\varepsilon$.

\section{Optimal control}

Now, we will introduce some control strategies, prevention and treatment. By including these controls into the model, our mathematical model is of the following form

$$
\frac{d P}{d t}=\alpha-\left(1-u_{1}\right) \beta P S-\gamma P,
$$

$\frac{d S}{d t}=\left(1-u_{1}\right) \beta P S-\delta\left(1-u_{2}\right) S-(\gamma+\varepsilon) S+\zeta\left(1-u_{1}\right) X$,

$$
\begin{aligned}
& \frac{d X}{d t}=\delta(1-\eta) S-\gamma X-\zeta\left(1-u_{1}\right) X \\
& \frac{d Y}{d t}=\delta \eta\left(1-u_{2}\right) S-\gamma Y \\
& \frac{d Z}{d t}=\varepsilon S-(\gamma+\vartheta) Z
\end{aligned}
$$

The parameter $u_{1}(t)$ represents the awareness campaign through the media transmission to control the potential smokers and temporary quit smokers to become smokers and $u_{2}(t)$ represents the treatment of smokers. Let us suppose that the objective functional $J$ is of the form

$J\left(u_{1}, u_{2}\right)=\int_{0}^{T}\left(A_{1} S+\frac{B_{1}}{2} u_{1}^{2}+\frac{B_{2}}{2} u_{2}^{2}\right) d t$,

where $A_{1}$ represents the positive weight. We will have to reduce the smokers and implementation charges of controls $u_{1}(t)$ and $u_{2}(t)$ with the aid of the above mentioned objective functional. We shall have the controls $u_{1}^{*}$ and $u_{2}^{*}$ such that

$J\left(u_{1}^{*}, u_{2}^{*}\right)=\min \left\{J\left(u_{1}, u_{2}\right),\left(u_{1}, u_{2}\right) \in U\right\}$,

where the set of controls $U$ is given below

$U=\left\{\left(u_{1}, u_{2}\right) \mid u_{i}(t)\right.$ is Lebsgue measurable on $[0,1]$,

$$
\left.0 \leq u_{i}(t) \leq 1, i=1,2\right\}
$$

In (Pontryagin et al., 1986), Pontryagin gave a principle for the solution of optimal control problem 
and deduction of mandatory conditions, this is called Pontryagin's Maximum Principle. We will make use of this principle.

\section{Existence of an optimal control}

To show that the optimal control problem exists, we need to check that our problem is satisfying some well-known hypotheses, these are stated in (Fleming \& Rishel, 1975) and given below :

$\left(H_{1}\right)$ The set of controls and corresponding state variables is nonempty.

$\left(H_{2}\right)$ The control set must be closed and convex.

$\left(H_{3}\right)$ The right hand side of the state system is bounded by a linear function in the state and control.

$\left(\mathrm{H}_{4}\right)$ The integrand of the objective functional $J$ is convex on $U$ and is bounded below by $c_{1}\left(\left|u_{1}\right|^{2}+\left|u_{2}\right|^{2}\right)^{\frac{\beta}{2}}-c_{2}$, where $c_{1}, c_{2}>0$ and $\beta>1$.

We will make use of a result, which is given by Lukes (Lukes, 1982, Th 9.2.1, p 182) to confirm that the solution of system (6) exists. In this manner, above hypothesis will be confirmed. $\left(H_{1}\right)$ is fulfilled because the coefficients are bounded. The boundedness of solutions tells that the set of controls $U$ fulfils the $2^{\text {nd }}$ hypotheses $\left(\mathrm{H}_{2}\right)$. By observing the system (6), we come to know the bi linearity of system in and, thus, the right hand side of (6) fulfils (because the boundedness of the solution of system). Positivity of integrand of the objective functional and bi linearity in controls imply convexity. $\left(H_{4}\right)$ is also satisfied

$A_{1} S+\frac{B_{1}}{2} u_{1}^{2}+\frac{B_{2}}{2} u_{2}^{2} \geq c_{1}\left(\left|u_{1}\right|^{2}+\left|u_{2}\right|^{2}\right)^{\frac{\beta}{2}}-c_{2}$,

where $c_{1}, c_{2}>0, A_{1}, B_{1}, B_{2}>0$ and $\beta>1$. Hence, we have the following theorem.

Theorem 8. For the system (6) the objective functional $\quad J\left(u_{1}, u_{2}\right)=\int_{0}^{T}\left(A_{1} S+\frac{B_{1}}{2} u_{1}^{2}+\frac{B_{2}}{2} u_{2}^{2}\right) d t$ where $U=\left\{\left(u_{1}, u_{2}\right) \mid 0 \leq u_{1}, u_{2} \leq 1, t \in[0, T]\right.$ subject to Eqs.(6) under some initial conditions, then an optimal control $u=\left(u_{1}^{*}, u_{2}^{*}\right)$ exists such that $J\left(u_{1}^{*}, u_{2}^{*}\right)=$ $\min \left\{J\left(u_{1}, u_{2}\right),\left(u_{1}, u_{2}\right) \in U\right\}$.

The optimal solution can be obtained by finding the Lagrangian and Hamiltonian for the optimal control problem (6). The Lagrangian of the optimal problem is defined as

$L\left(S, u_{1}, u_{2}\right)=A_{1} S+\frac{B_{1}}{2} u_{1}^{2}+\frac{B_{2}}{2} u_{2}^{2}$.
The minimal value of the Lagrangian can be obtained by defining the Hamiltonian. Let us take $X=(P, S, X, Y, Z), U=\left(u_{1}, u_{2}\right) \quad$ and $\lambda=\left(\lambda_{1}, \lambda_{2}, \lambda_{3}, \lambda_{4}, \lambda_{5}\right)$, to obtain:

$H(X, U, \lambda)=L\left(S, u_{1}, u_{2}\right)+\lambda_{1}\left(\alpha-\left(1-u_{1}\right) \beta P S-\gamma P\right)$

$+\lambda_{2}\left(\left(1-u_{1}\right) \beta P S-\delta\left(1-u_{2}\right) S-(\gamma+\varepsilon) S+\right.$

$\left.\zeta\left(1-u_{1}\right) X\right)+\lambda_{3}\left(\delta(1-\eta) S-\gamma X-\zeta\left(1-u_{1}\right) X\right)+$

$\lambda_{4}\left(\delta \eta\left(1-u_{2}\right) S-\gamma Y\right)+\lambda_{5}(\varepsilon S-(\gamma+\vartheta) Z)$.

\section{The optimality system}

We invoke Pontryagin's Maximum Principle (Lenhart \& Workman, 2007), for finding the mandatory conditions for this optimal control, as follows: If the solution of control problem is $\left(u_{1}^{*}, u_{2}^{*}\right)$ then we have a nontrivial vector function $\lambda(t)=\left(\lambda_{1}(t), \lambda_{2}(t), \ldots, \lambda_{5}(t)\right)$ such that the following conditions are satisfied. The state equation is

$\frac{d x}{d t}=\frac{\partial H\left(t, u_{1}^{*}, u_{2}^{*}, \lambda(t)\right)}{\partial \lambda}$,

the condition of optimality

$$
=\frac{\partial H\left(t, u_{1}^{*}, u_{2}^{*}, \lambda(t)\right)}{\partial u},
$$

and adjoint equation

$\frac{d \lambda}{d t}=-\frac{\partial H\left(t, u_{1}^{*}, u_{2}^{*}, \lambda(t)\right)}{\partial x}$

Now, the mandatory conditions will be applied to the Hamiltonian $H$.

Theorem 9. For the optimal controls $u_{1}^{*}, u_{2}^{*}$ and the solutions $P^{*}, S^{*}, X^{*}, Y^{*}, Z^{*}$ of the system (6), we have adjoint variables $\lambda_{1}, \lambda_{2}, \ldots, \lambda_{5}$ which satisfy

$\frac{d \lambda_{1}}{d t}=\left(\lambda_{1}-\lambda_{2}\right) \beta S+\lambda_{1} \gamma$,

$$
\begin{aligned}
\frac{d \lambda_{2}}{d t} & =-A_{1}+\left(\lambda_{1}-\lambda_{2}\right)\left(1-u_{1}\right) \beta P+\lambda_{2} \delta\left(1-u_{2}\right) \\
& +\lambda_{2}(\gamma+\varepsilon)-\lambda_{3} \delta(1-\eta)-\lambda_{4} \delta \eta\left(1-u_{2}\right)-\lambda_{5} \varepsilon,
\end{aligned}
$$

$\frac{d \lambda_{3}}{d t}=-\lambda_{2} \zeta\left(1-u_{1}\right)+\lambda_{3} \gamma+\lambda_{3} \zeta\left(1-u_{1}\right)$, 
$\frac{d \lambda_{4}}{d t}=\lambda_{4} \gamma$

$\frac{d \lambda_{5}}{d t}=\lambda_{5}(\gamma+\vartheta)$

with the conditions of transversality $\lambda_{1}(T)=\lambda_{2}(T)=\lambda_{3}(T)=\lambda_{4}(T)=\lambda_{5}(T)=0$.

Moreover, $u_{1}^{*}, u_{2}^{*}$ will be given by

$u_{1}^{*}=\max \left\{\min \left\{1, \frac{1}{B_{1}}\left(\left(\lambda_{2}-\lambda_{1}\right) \beta P S+\left(\lambda_{2}-\lambda_{3}\right) \zeta X\right)\right\}, 0\right\}$,

$u_{2}^{*}=\max \left\{\min \left\{1, \frac{1}{B_{2}}\left(\lambda_{4} \delta \eta S-\lambda_{2} \delta S\right)\right\}, 0\right\}$.

Proof. Hamiltonian $H$ will be used to obtain the adjoint equations and the conditions of transversality. Now, take the derivative of $H$ w. r. t. $P, S, X, Y, Z$ and putting $P=P^{*}, S=S^{*}, X=X^{*}, Y=Y^{*}, Z=Z^{*}$, we have the following equations

$$
\begin{aligned}
\frac{d \lambda_{1}}{d t}= & \left(\lambda_{1}-\lambda_{2}\right) \beta S+\lambda_{1} \gamma, \\
\frac{d \lambda_{2}}{d t}= & -A_{1}+\left(\lambda_{1}-\lambda_{2}\right)\left(1-u_{1}\right) \beta P+\lambda_{2} \delta\left(1-u_{2}\right)+ \\
& \lambda_{2}(\gamma+\varepsilon)-\lambda_{3} \delta(1-\eta)-\lambda_{4} \delta \eta\left(1-u_{2}\right)-\lambda_{5} \varepsilon, \\
\frac{d \lambda_{3}}{d t}= & -\lambda_{2} \zeta\left(1-u_{1}\right)+\lambda_{3} \gamma+\lambda_{3} \zeta\left(1-u_{1}\right), \\
\frac{d \lambda_{4}}{d t}= & \lambda_{4} \gamma, \\
\frac{d \lambda_{5}}{d t}= & \lambda_{5}(\gamma+\vartheta),
\end{aligned}
$$

with the conditions of transversality $\lambda_{1}(T)=\lambda_{2}(T)=\lambda_{3}(T)=\lambda_{4}(T)=\lambda_{5}(T)=0 . \quad$ By the use of optimality conditions and the property of the control space $U$, we can have the following form of controls $u_{1}^{*}, u_{2}^{*}$,

$$
\begin{aligned}
& u_{1}^{*}=\max \left\{\min \left\{1, \frac{1}{B_{1}}\left(\left(\lambda_{2}-\lambda_{1}\right) \beta P S+\left(\lambda_{2}-\lambda_{3}\right) \zeta X\right)\right\}, 0\right\}, \\
& u_{2}^{*}=\max \left\{\min \left\{1, \frac{1}{B_{2}}\left(\lambda_{4} \delta \eta S-\lambda_{2} \delta S\right)\right\}, 0\right\} .
\end{aligned}
$$

Outcome of the optimal control strategies on the transmission of smoking disease can also be observed numerically. In Figure 6a, we observe that the endemic level of potential smokers increases by applying the control. It seems true because practically if the smokers have been removed from the community then total individuals will be non-smokers and enter into the potential smokers' class. As a result, potential smokers increase. Figure $6 \mathrm{~b}$ shows the behaviour of smokers with and without control. We can see interesting behaviour of smokers with control. After $\sim 120$ days, smokers start to increase even though the control is being applied consistently. After $\sim 300$ days, smokers start to decline and become zero after 400 days. According to the graph, there will be no more smoker present in the community after 400 days by the application of these controls. Control profile is shown in Figure 6c. From this figure, we can see that the control $U_{2}$ remains zero till 500 days. It represents the treatment of smokers by using the injection containing drugs that block nicotine receptors of the brain which in turn reduces the desire to smoke. But we can see that this strategy play no role in reducing the number of smokers. However, the education campaign is effective in reducing the number of smokers from the community.

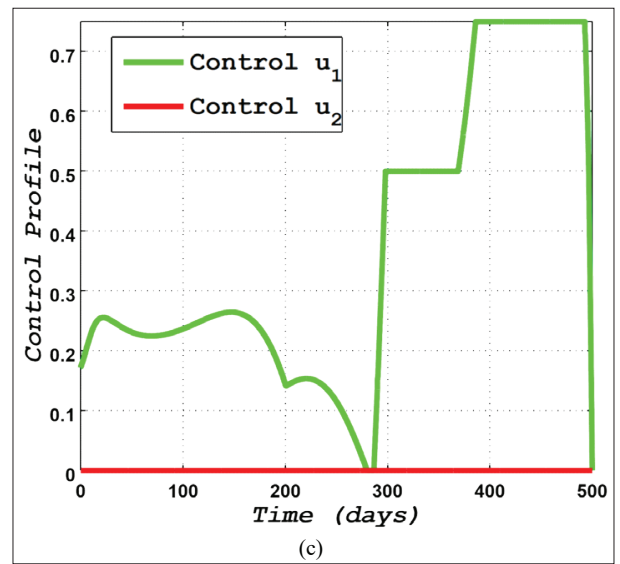

Figure 6: Variation in the number of Potential smokers and smokers by the application of controls.

\section{Nonstandard difference scheme}

In this section, we apply a nonstandard difference scheme (Mickens, 2002) to system of equations (1-5) as follows:

$$
\begin{aligned}
P_{n+1} & =\frac{h \alpha+P_{n}}{1+h \beta S_{n}+h \gamma}, \\
S_{n+1} & =\frac{S_{n}+h \beta P_{n} S_{n}+h \zeta X_{n}}{1+h(\gamma+\delta+\varepsilon)},
\end{aligned}
$$




$$
\begin{gathered}
X_{n+1}=\frac{X_{n}+h \delta(1-\eta) S_{n}}{1+h(\gamma+\zeta)}, \\
Y_{n+1}=\frac{Y_{n}+h \delta \eta S_{n}}{1+h \gamma}, \\
Z_{n+1}=\frac{Z_{n}+h \varepsilon S_{n}}{1+h(\gamma+\vartheta)},
\end{gathered}
$$

where $h$ is the step size. Then, it is easy to see that nonstandard finite difference scheme implemented in discrete system (7) is positivity and steady-states preserving scheme. Indeed, the equilibria of (7) are computed as follows in closed forms:
$E_{0}=\left(\frac{\alpha}{\gamma}, 0,0,0,0\right)$

and

$$
E^{*}=\left(P^{*}, S^{*}, X^{*}, Y^{*}, Z^{*}\right)
$$

where

$$
\begin{aligned}
P^{*}: & =\frac{\gamma^{2}+\gamma(\delta+\varepsilon+\zeta)+\zeta(\varepsilon+\delta \eta)}{\beta(\gamma+\zeta)}, \\
S^{*} & :=-\frac{\gamma}{\beta}+\frac{\alpha(\gamma+\zeta)}{\gamma^{2}+\gamma(\delta+\varepsilon+\zeta)+\zeta(\varepsilon+\delta \eta)},
\end{aligned}
$$

$$
\begin{aligned}
X^{*}:=\frac{\delta(\eta-1)\left(-\alpha \beta(\gamma+\zeta)+\gamma\left(\gamma^{2}+\gamma(\delta+\varepsilon+\zeta)+\zeta(\varepsilon+\delta \eta)\right)\right)}{\beta(\gamma+\zeta)\left(\gamma^{2}+\gamma(\delta+\varepsilon+\zeta)+\zeta(\varepsilon+\delta \eta)\right)}, \\
Y^{*}:=-\frac{\delta \eta\left(-\alpha \beta(\gamma+\zeta)+\gamma\left(\gamma^{2}+\gamma(\delta+\varepsilon+\zeta)+\zeta(\varepsilon+\delta \eta)\right)\right)}{\beta \gamma\left(\gamma^{2}+\gamma(\delta+\varepsilon+\zeta)+\zeta(\varepsilon+\delta \eta)\right)},
\end{aligned}
$$

and

$$
Z^{*}:=-\frac{\varepsilon\left(-\alpha \beta(\gamma+\zeta)+\gamma\left(\gamma^{2}+\gamma(\delta+\varepsilon+\zeta)+\zeta(\varepsilon+\delta \eta)\right)\right)}{\beta\left(\gamma^{2}+\gamma(\delta+\varepsilon+\zeta)+\zeta(\varepsilon+\delta \eta)\right)(\gamma+\vartheta)}
$$

Then $E^{*}$ be unique positive equilibrium point of system (7) if and only if $\frac{\gamma}{\beta}<\frac{\alpha(\gamma+\zeta)}{\gamma^{2}+\gamma(\delta+\varepsilon+\zeta)+\zeta(\varepsilon+\delta \eta)}$. Now, Jacobian matrix for (7) computed at $E_{0}$ is given as follows:

$$
J\left(E_{0}\right)=\left(\begin{array}{ccccc}
\frac{1}{1+h \gamma} & -\frac{h \alpha \beta}{\gamma+h \gamma^{2}} & 0 & 0 & 0 \\
0 & \frac{1+\frac{h \alpha \beta}{\gamma}}{1+h(\gamma+\delta+\varepsilon)} & \frac{h \zeta}{1+h(\gamma+\delta+\varepsilon)} & 0 & 0 \\
0 & \frac{h \delta(1-\eta)}{1+h(\gamma+\zeta)} & \frac{1}{1+h(\gamma+\zeta)} & 0 & 0 \\
0 & \frac{h \delta \eta}{1+h \gamma} & 0 & \frac{1}{1+h \gamma} & 0 \\
0 & \frac{h \varepsilon}{1+h(\gamma+\vartheta)} & 0 & 0 & \frac{1}{1+h(\gamma+\vartheta)}
\end{array}\right)
$$

The characteristic polynomial for $J\left(E_{0}\right)$ is given as follows:

$P_{1}(\lambda)=\left(\lambda-\frac{1}{1+h \gamma}\right)^{2}\left(\lambda-\frac{1}{1+h(\gamma+\vartheta)}\right)\left(\lambda^{2}-\left(a_{1}+a_{4}\right) \lambda+a_{1} a_{4}-a_{2} a_{3}\right)$, 
where

$$
\begin{aligned}
& a_{1}:=\frac{1+\frac{h \alpha \beta}{\gamma}}{1+h(\gamma+\delta+\varepsilon)}, a_{2}:=\frac{h \zeta}{1+h(\gamma+\delta+\varepsilon)}, \\
& a_{3}:=\frac{h \delta(1-\eta)}{1+h(\gamma+\zeta)}, a_{4}:=\frac{1}{1+h(\gamma+\zeta)} .
\end{aligned}
$$

Due to aforementioned computation, we have the following Lemma.

Lemma 10. $E_{0}$ is a sink if and only if the following condition is satisfied:

$a_{1}+a_{4}<1+a_{1} a_{4}-a_{2} a_{3}<2$.

Similarly, the Jacobian matrix of (7) computed at $E^{*}$ is given as follows:

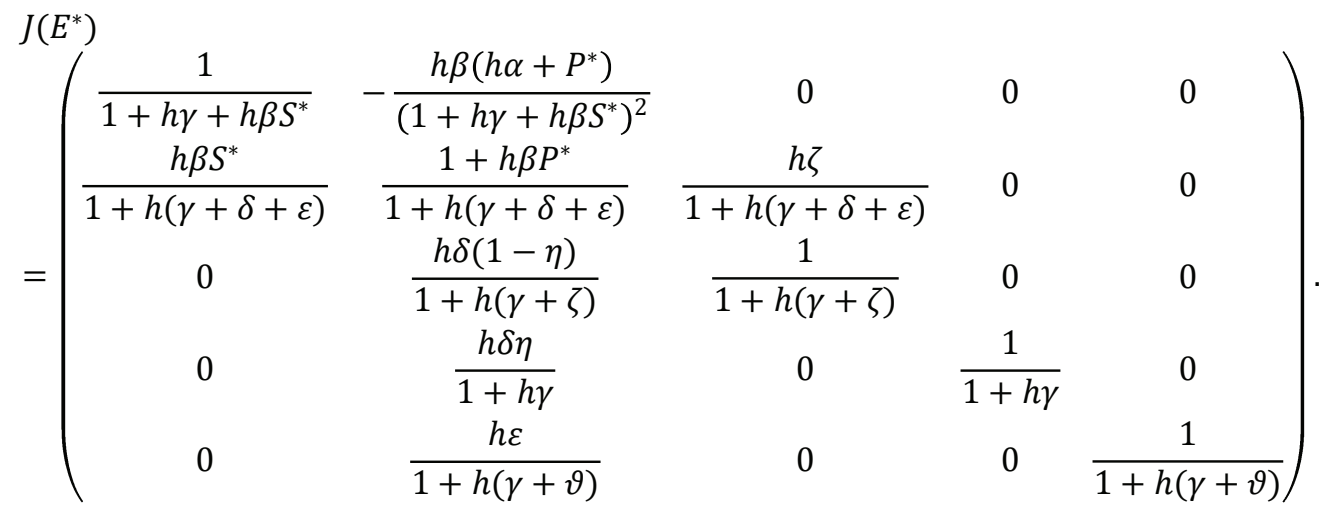

Moreover, the characteristic polynomial for $J\left(E^{*}\right)$ are computed as follows:

$$
P_{2}(\lambda)=\left(\lambda-\frac{1}{1+h \gamma}\right)\left(\lambda-\frac{1}{1+h(\gamma+\vartheta)}\right) Q(\lambda)
$$

where

$$
\begin{aligned}
& Q(\lambda):=\lambda^{3}-\left(b_{1}+b_{4}+b_{7}\right) \lambda^{2}+\left(b_{1} b_{4}-b_{2} b_{3}-b_{5} b_{6}+b_{1} b_{7}+b_{4} b_{7}\right) \lambda+b_{1} b_{5} b_{6}+b_{2} b_{3} b_{7}-b_{1} b_{4} b_{7}, \\
& b_{1}:=\frac{1}{1+h \gamma+h \beta S^{*}}, b_{2}:=-\frac{h \beta\left(h \alpha+P^{*}\right)}{\left(1+h \gamma+h \beta S^{*}\right)^{2}}, b_{3}:=\frac{h \beta S^{*}}{1+h(\gamma+\delta+\varepsilon)} \\
& b_{4}:=\frac{1+h \beta P^{*}}{1+h(\gamma+\delta+\varepsilon)}, b_{5}:=\frac{h \zeta}{1+h(\gamma+\delta+\varepsilon)}, b_{6}:=\frac{h \delta(1-\eta)}{1+h(\gamma+\zeta)}, b_{7}:=\frac{1}{1+h(\gamma+\zeta)}
\end{aligned}
$$

The following Lemma gives necessary and sufficient conditions that all roots of (9) lie inside the unit open disk.

Lemma 11. Assume that $\frac{\gamma}{\beta}<\frac{\alpha(\gamma+\zeta)}{\gamma^{2}+\gamma(\delta+\varepsilon+\zeta)+\zeta(\varepsilon+\delta \eta)}$, then unique positive equilibrium point $E^{*}$ is a sink if and only if

$$
\begin{aligned}
& \left|\alpha_{2}+\alpha_{0}\right|<1+\alpha_{1},\left|\alpha_{2}-3 \alpha_{0}\right|<3-\alpha_{1}, \text { and } \\
& \alpha_{0}^{2}+\alpha_{1}-\alpha_{0} \alpha_{2}<1,
\end{aligned}
$$

where

$\alpha_{2}:=-\left(b_{1}+b_{4}+b_{7}\right), \alpha_{1}:=b_{1} b_{4}-b_{2} b_{3}-b_{5} b_{6}$

$+b_{1} b_{7}+b_{4} b_{7}, \alpha_{0}:=b_{1} b_{5} b_{6}+b_{2} b_{3} b_{7}-b_{1} b_{4} b_{7}$

In order to show that proposed nonstandard difference scheme (NDS) in system (7) is dynamically consistent, we choose $\alpha=3.6, \quad \beta=0.04, \gamma=0.1, \quad \delta=0.3$, $\eta=0.04, \zeta=0.5, \varepsilon=0.1$ and $\vartheta=0.8$ for both systems (1) and (7) with initial conditions $\left(P_{0}, S_{0}, X_{0}, Y_{0}, Z_{0}\right)=$ $(6.4,11.2,5.4,1.35,1.2)$. Furthermore, implicit Runge 


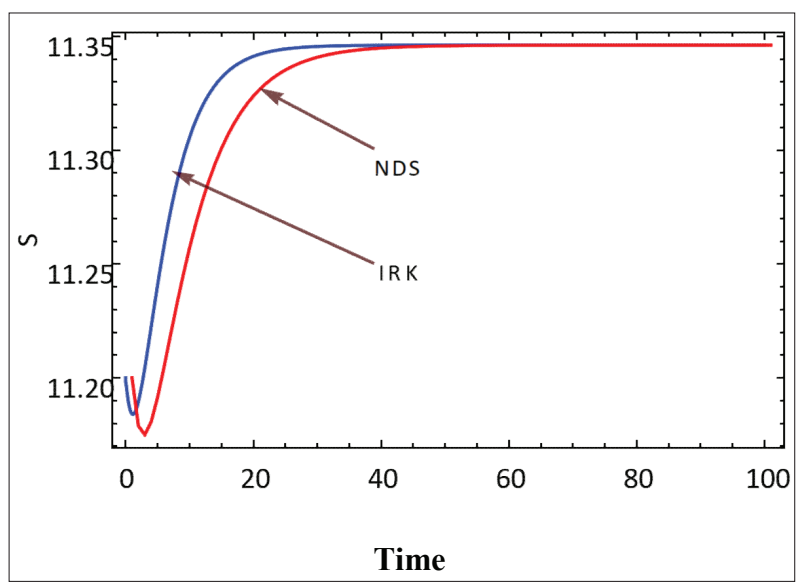

Figure 7: Plots of $P$ for system (1) (blue) and for system (7) (red).

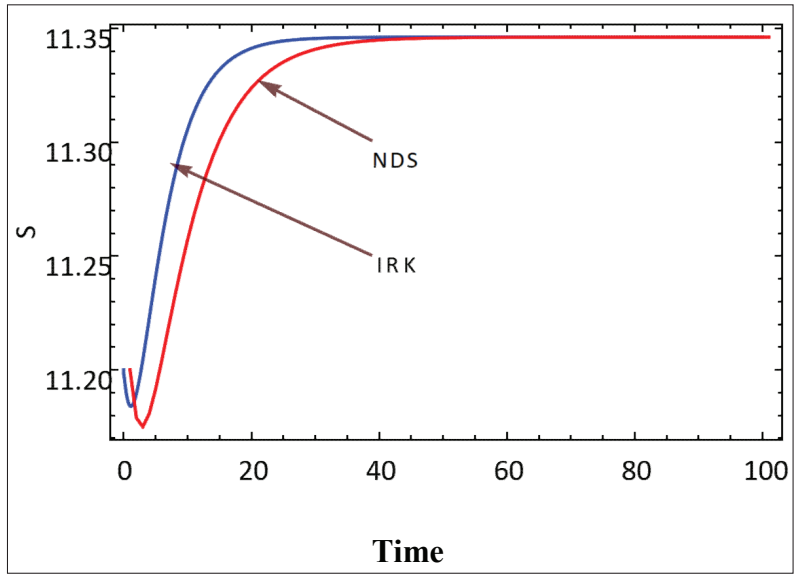

Figure 8: Plots of $S$ for system (1) (blue) and for system (7) (red)

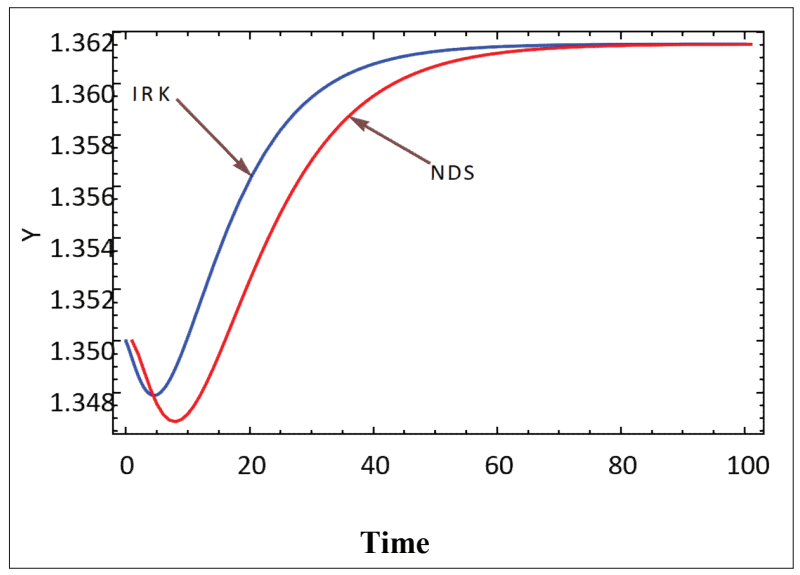

Figure 9: Plots of $X$ for system (1) (blue) and for system (7) (red).

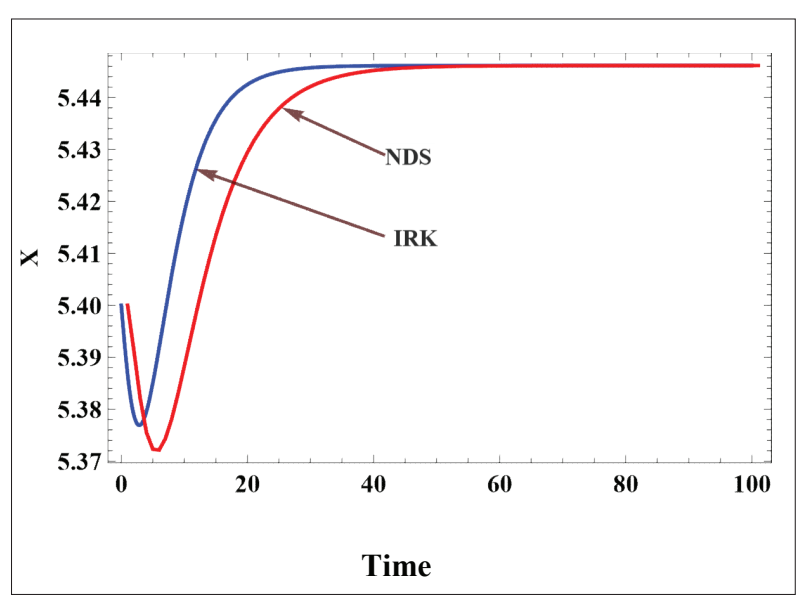

Figure 10: Plots of $X$ for system (1) (blue) and for system (7) (red).

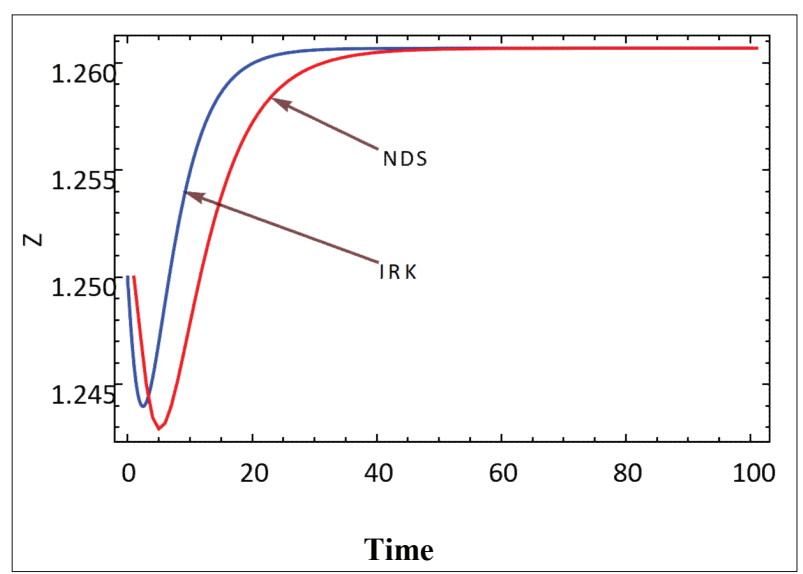

Figure 11: Plots of $Z$ for system (1) (blue) and for system (7) (red).

Kutta (IRK) method of order 4 with step size 0.0001 is implemented to the system (1) and $h$ is taken as 0.95 in system (7). Then plots for system of equations (1-5) (blue) and for system (7) (red) are depicted in the figures given below. The horizontal axis is the time in days.

\section{CONCLUSIONS}

In this paper, we presented and analysed the dynamics of mathematical model of smoking. This model contains five classes namely potential smokers, smokers, temporary quitters, permanent quitters and those individuals that have some disease as a result of smoking. We assumed that temporary quitters become smokers again. Some smokers suffer from diseases like lung cancer, mouth cancer and TB etc. So we have considered this class in 
the model. Evidences show that some people die due to smoking related diseases so disease related death rate in the model has also been assumed. We have made use of matrix theoretic method to show that smoke free equilibrium point is globally stable for $R_{0}<1$. It is shown that for $R_{0}>1$, the endemic point persists and its global stability has been studied with the help of graph theoretic approach. The sensitivity analysis of threshold parameter $R_{0}$ and smokers is discussed and on the basis of this sensitivity analysis an optimal control strategy has been established. Two kinds of controls strategies have been introduced, one is related with the awareness campaign through the media transmission to control the potential smokers and interimly quit smokers to become smokers and the $2 n d$ is treatment of smokers. With the help of numerical simulations effect of these proposed controls have been studied. The outcomes tell that these two control strategies are very effective in reducing the endemic level of smokers. At the end, a dynamically consistent, positivity and fixed points conserving nonstandard difference scheme is implemented. Since exact solutions of system of equations (1-5) can not be obtained in closed form. In order to compare proposed nonstandard difference scheme (7) with its continuous counterpart (1-5) higher-order implicit Runge--Kutta method with smaller step size is implemented.

\section{REFERENCES}

Anderson R. M. \& May R. M. (1982). Population Biology of Infectious Diseases, Springer Verlag, Berlin, Heidelberg, Germany.

Anderson R. M. \& May R. M. (1991). Infectious Diseases of Humans, Oxford University Press, London, UK.

Awan A., Sharif A., Hussain T. \& Ozair M. (2017). Smoking model with cravings to smoke. Advanced Studies in Biology 9(1): 31-41. DOI: https://doi.org/10.12988/asb.2017.61245

Bernoulli D. (1760). Essai d'une nouvelle analyse de la mortalit cause par la petite vrole, et des avantages de l'inoculation pour la prvenir, pp. 1-45. Histoire de l'Academie Royale des Sciences, Paris., 1-45.

Brauer F. \& Castillo C. C. (2001). Mathematical Models in Population Biology and Epidemiology, Springer, Berlin, Germany.

Castillo G. C., Jordan S. G. \& Herrera A. R. (1997). Mathematical models for the dynamics of tobacco use, recovery, and relapse, Technical Report Series BU-1505-M, Cornell University, Ithaca, NY, USA. DOI: https://doi.org/10.1186/s13662-016-0830-6

Driessche P. V. D. \& Watmough J. (2002). Reproduction numbers and sub-threshold endemic equilibria for compartmental models of disease transmission.
Mathematical biosciences 180: 29-28. DOI:https://doi.org/10.1016/S0025-5564(02)00108-6

Fleming W. H. \& Rishel R. W. (1975). Deterministic and Stochastic Optimal Control, Springer, New York, USA.

Harary F. (1969). Graph Theory. Addison-Wesley, Reading, MA.

Johnston L. D., O’Malley P. M. \& Bachman J. G. (1992). Smoking, Drinking, and fllicit Drug Use among American Secondary School Students, College Students, and Young Adults, 1975-1992. Volume 2: College Students and Young Adults. Bethesda, Md: US Department of Health and Human Services, National Institutes of Health; NIH publication 93-3481.

Kermack W. O. \& Mckendrick A. G. (1927). A contributions to the Mathematical Theory of Epidemics. Part I, Proceeding of Royal Society of London Series A 115: 700-721.

DOI:https://doi.org/10.1098/rspa.1927.0118

Kermack W. O. \& Mckendrick A. G. (1932). Contributions to the Mathematical Theory of Epidemics. Part II, Proceeding of Royal Society of London Series A 138: 55-83.

DOI:https://doi.org/10.1098/rspa.1932.0171

Kermack W. O. \& Mckendrick A. G. (1933). Contributions to the Mathematical Theory of Epidemics. Part III, Proceeding of Royal Society of London Series B 141: 94-112.

DOI:https://doi.org/10.1098/rspa.1933.0106

Lahrouz A., OmariL L., Kiouach D. \& BelmaÃ ti A. (2011). Deterministic and stochastic stability of a mathematical model of smoking, Statistics and Probability Letters 81: 1276-1284.

DOI:https://doi.org/10.1016/j.spl.2011.03.029

LaSalle J. P. (1976). The Stability Of Dynamical Systems, Society for Industrial and Applied Mathematics, Philadelphia, Pennsylvania, USA.

DOI:https://doi.org/10.1137/1.9781611970432

Lenhart S. \& Workman J. T. (2007). Optimal Control Applied To Biological Models. Mathematical and Computational Biology Series Chapman and Hall/CRC Press,London/ Boca Raton.

Lukes D. L. (1982). Differential equations: Classical to Controlled, Mathematics in Science and Engineering, Academic Press, New York, USA.

Mickens R. E. (2002). Nonstandard finite difference schemes for differential equations. Journal of Difference Equations and Applications 8(9): 823-47. DOI: https://doi.org/10.1080/1023619021000000807

Pontryagin L.S., Boltyanskii V.G., Gamkrelidze R. V. \& Mishchenko E.F. (1986). The Mathematical Theory of Optimal Processes, volume 4, Gordon and Breach Science Publishers, New York, NY, USA. DOI:https://doi.org/10.1186/s12889-018-5388-7

Shaheen K., Oyebode O. \& Masud H. (2018). Experiences of young smokers in quitting smoking in twin cities of Pakistan: a phenomenological study. BMC Public Health 18(1): 466.

Sharomi O. \& Gumel A. B. (2008). Curtailing smoking dynamics: a mathematical modeling approach. 
Applied Mathematics and Computation 195: 475-499. DOI:https://doi.10.1016/j.amc.2007.05.012

Shuai Z. \& Driessche P. V. D. (2013). Global stability of infectious disease models using Lyapunov functions. SIAM Journal on Applied Mathematics 73: 1513-1532. DOI:https://doi.org/10.1137/120876642

Verma V. \& Agarwal M. (2015). Global dynamics of a mathematical model on smoking with media campaigns. Research Desk 4(1): 500-512.

Verma V. \& Bhadauria A. (2019). Global dynamics of a mathematical model on smoking: impact of antismoking campaign. Journal of Mathematical Modeling 7(1): 49-62. DOI:https://10.22124/JMM.2018.10117.1153

West D. B. (1996). Introduction to Graph Theory, PrenticeHall, Upper Saddle River, NJ.

World Health Organization. (2017). WHO Report On The
Global Tobacco Epidemic, 2017: Monitoring Tobacco Use And Prevention Policies. World Health Organization, Geneva, Switzerland.

World Health Organization. (2009). WHO report on the global tobacco epidemic, 2009. World Health Organization.

Zaman G. (2011). Qualitative behavior of giving up smoking model. Bulletin of the Malaysian Mathematical Sciences Society 34: 403-415.

Zeb A., Zaman G. \& Momani S. (2012). Squareroot Dynamics of a Giving Up Smoking Model. Applied Mathematical Modelling 37: 5326-5334. DOI:https://doi.org/10.1016/j.apm.2012.10.005

Zeb A., Bano A., Alzahrani E. \& Zaman G. (2018). Dynamical analysis of cigarette smoking model with a saturated incidence rate. AIP Advances 8(4): 045317. DOI: https://doi.org/10.1063/1.5028157 